Cahiers « Mondes anciens »

ANCIENS

Histoire et anthropologie des mondes anciens

$5 \mid 2014$

Maudire et mal dire : paroles menaçantes en Grèce ancienne

\title{
Au nom du père : la malédiction paternelle en Grèce ancienne
}

Jean-Baptiste Bonnard

\section{CpenEdition}

Journals

Édition électronique

URL : http://journals.openedition.org/mondesanciens/1239

DOI : 10.4000/mondesanciens.1239

ISSN : 2107-0199

Éditeur

UMR 8210 Anthropologie et Histoire des Mondes Antiques

Référence électronique

Jean-Baptiste Bonnard, "Au nom du père : la malédiction paternelle en Grèce ancienne », Cahiers

«Mondes anciens » [En ligne], 5| 2014, mis en ligne le 14 février 2014, consulté le 14 novembre 2019.

URL : http://journals.openedition.org/mondesanciens/1239; DOI : 10.4000/mondesanciens.1239

Ce document a été généré automatiquement le 14 novembre 2019.

\section{cc) (1)}

Les Cahiers «Mondes Anciens » sont mis à disposition selon les termes de la licence Creative Commons Attribution - Pas d'Utilisation Commerciale - Pas de Modification 4.0 International. 


\title{
Au nom du père : la malédiction paternelle en Grèce ancienne
}

\author{
Jean-Baptiste Bonnard
}

1 Le terme «malédiction » a usuellement deux sens intimement liés. Il désigne d'abord les paroles par lesquelles on souhaite avec véhémence tout le mal possible à un individu ou à un groupe (une famille, une cité...), en appelant implicitement ou explicitement le courroux des puissances divines ou d'une instance supérieure (le sort, le destin...) à intervenir au service de ce souhait. De ce premier sens du terme, auquel on pourrait réserver le synonyme, moins polysémique, d'«imprécation », découle un prolongement qui n'est que la traduction ou l'accompagnement en actes des paroles d'imprécations: ainsi, une malédiction peut être, éventuellement, une pratique rituelle destinée à assurer l'efficacité du souhait. Le deuxième sens désigne l'effet des imprécations et des éventuelles pratiques malédictives sur celui ou ceux contre qui elles ont été lancées ou mises en œuvre: dans ce sens, on parle de malédiction pour désigner le malheur inéluctable qui pèse sur celui qui en est victime (la malédiction est donc aussi le fait d'être maudit) et la réalisation effective de l'imprécation ${ }^{1}$. Ces deux sens nous intéressent et plus particulièrement le premier. En grec, un terme désigne la malédiction, surtout dans ce premier sens, d'imprécation, celui d'ara qui exprime, de façon générale, la notion de demande aux dieux par une prière, mais qui s'est spécialisé, surtout au pluriel (arai), pour désigner l'imprécation, la malédiction lancée contre quelqu'un. L'étymologie en est incertaine: Antoine Meillet a fait le rapprochement avec un groupe de mots qui se retrouvent dans plusieurs langues indo-européennes pour désigner le fait de prononcer des paroles de façon solennelle ${ }^{2}$.

2 Les sociétés grecques, comme beaucoup d'autres, connaissent la pratique de la malédiction qui semble donc avoir une dimension structurelle anthropologique. Les malédictions qui vont retenir notre attention sont celles lancées, dans les mythes grecs, par des pères contre des fils. Nous étudierons plus précisément trois cas pour lesquels nous sommes particulièrement renseignés. Nous commencerons par présenter les trois malédictions qui nous intéressent avant d'en mettre en évidence les spécificités. 


\section{Les malédictions paternelles lancées par Erdipe, Thésée et Amyntor: les sources}

\section{Edipe}

3 La malédiction pour laquelle nous avons le plus de sources est celle lancée par đedipe contre ses deux fils Étéocle et Polynice. C'est sans doute par les Phéniciennes que cette malédiction a acquis sa notoriété - en tout cas sa notoriété moderne. Mais Euripide n'est assurément pas l'inventeur du motif. Quelle est la trace la plus ancienne de cet épisode? Il n'en est pas question chez Homère ${ }^{3}$. Dans la geste thébaine du Cycle épique, deux poèmes pouvaient le traiter, l'Edipodie et la Thébaïde sujet la vie d'Ædipe, comme l'indique assez son titre, le second ${ }^{5}$ parce que, narrant la guerre des Sept contre Thèbes, il devait faire état de la malédiction lancée contre les deux fils d'ÆEdipe, cause lointaine de cette guerre, sans nécessairement en constituer la matière essentielle comme le pense Walter Burkert ${ }^{6}$. Dans les malheureux débris conservés de l' Edipodie, nous n'en pouvons trouver de traces. La présence de cet épisode dans le résumé que donne Albert Séveryns de cette épopée est donc purement conjecturale, mais assez probable dans la mesure où ce poème est, pense-t-on ordinairement, un remaniement de la Thébaïde et que celle-ci était sans doute alors très populaire, ce qui rendait difficile d'en supprimer un élément aussi important ${ }^{7}$. Or, des fragments de la Thébaïde, qui est un peu moins naufragée que l'œdipodie, deux attestent précisément cette malédiction. Le fragment 2, rapporté par Athénée dit en effet :

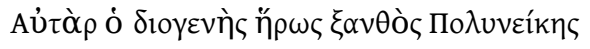

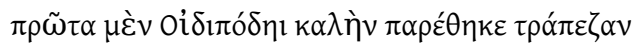

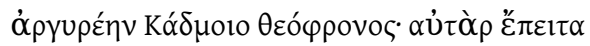

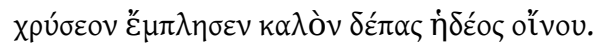

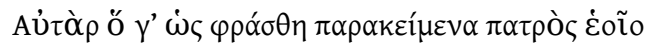

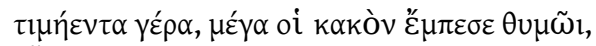

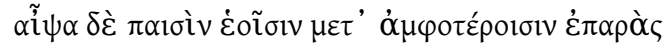

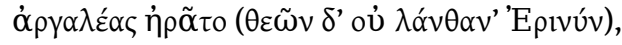

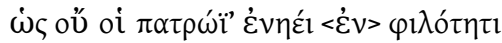

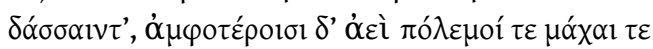

Mais le héros issu des dieux, le blond Polynice, d'abord devant Ædipe plaça une belle table d'argent, celle de Kadmos inspiré des dieux ; ensuite il emplit une coupe d'or d'un doux vin. Mais @edipe, dès qu'il eut remarqué devant lui les dons précieux de son père, une grande colère lui tomba au cœur, et aussitôt il maudissait gravement ses deux fils (et cette malédiction n'échappe pas à l'Érinys), souhaitant qu'ils ne partagent pas dans une amitié fraternelle les dons de leur père, mais qu'entre eux toujours il y ait guerres et combats 8 .

Le fragment 3, conservé par une scholie au vers 1375 de l'œdipe à Colone, indique :

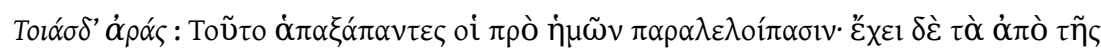

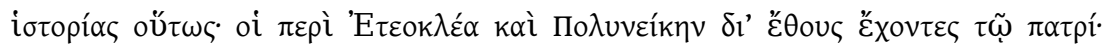

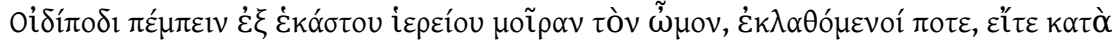

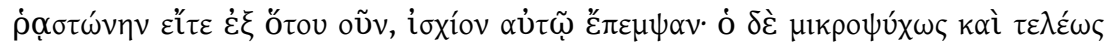

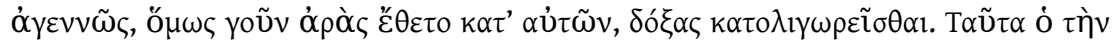

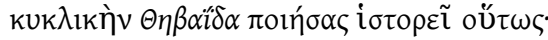

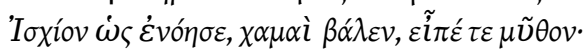

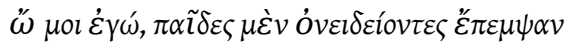




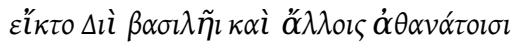

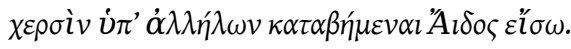

«Ces imprécations » : Tous ceux qui m'ont précédé ont négligé ce point. Les détails de l'histoire sont les suivants. Étéocle et Polynice avaient l'habitude d'envoyer comme part à leur père đdipe l'épaule de chacune des victimes. Mais un jour, à leur insu, par insouciance ou pour toute autre raison, ils lui envoyèrent la hanche. Alors đdipe, parce qu'il lui semblait qu'on le négligeait, lança contre eux également, bassement et irrévocablement, des imprécations. L'auteur de la Thébaïde les décrit de la manière suivante: Dès qu'il comprit que c'était une hanche, il la jeta à terre et prononça ces paroles: « Hélas, mes enfants me l'ont envoyée pour m'outrager... Plaise au roi Zeus et aux autres immortels de les faire descendre dans l'Hadès sous les coups l'un de l'autre $»^{9}$.

Laissons provisoirement de côté les problèmes que posent ces deux textes, et notamment leur articulation, et examinons les autres sources. Puisque les poètes lyriques n'ont apparemment pas traité ce thème ${ }^{10}$, bien que certains d'entre eux (notamment les Béotiens Corinne et Pindare, mais aussi peut-être Mimnerme) se soient intéressés à la geste thébaine, tournons-nous vers les Tragiques. Les Sept contre Thèbes est la troisième pièce, et seule survivante, d'une trilogie d'Eschyle représentée en 467 . Du Laios et de l' Edipe qui la précédaient ne nous est à peu près rien parvenu (aucun fragment sûr du Laios ; un seul fragment de l'(Edipe), mais les vers 742-757 et 766-791 des Sept permettent d'avoir une idée de leur contenu ${ }^{11}$. La malédiction paternelle était vraisemblablement lancée dans l'‘dipe ${ }^{12}$. Les vers des Sept où Étéocle parle d'un cauchemar qu'il a eu y font sans doute allusion :

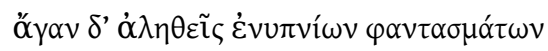

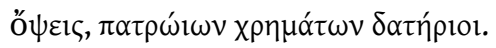

Elles n'étaient que trop vraies, les visions de mes songes, qui partageaient mon patrimoine $e^{13}$ !

Dans cette troisième pièce, le motif de la malédiction est présent tout du long ${ }^{14}$, ce qui a d'ailleurs conduit un commentateur moderne, Friedrich Solmsen, à écrire qu'elle en était le leitmotiv ${ }^{15}$. Sophocle fait probablement allusion à la malédiction lancée par đEdipe sur ses fils, dans la plus ancienne de ses pièces conservées, Antigone (442). Au vers 603, à propos des malheurs qui frappent la race d'€Edipe en général et plus particulièrement de

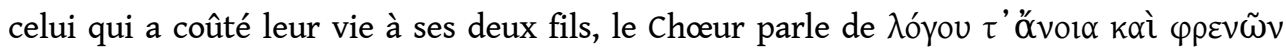
'Epivúc «la folie d'un discours et de l'Érinys d'une pensée », ce qui nous paraît une

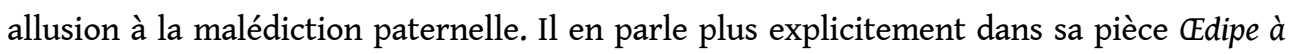
Colone jouée en 401 , aux vers 421 et suivants :

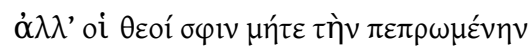

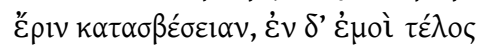

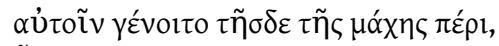

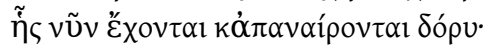

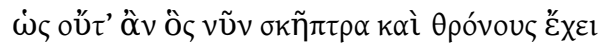

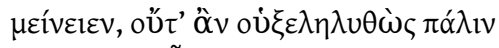

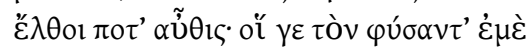

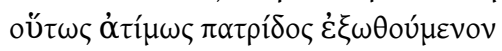

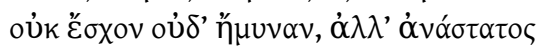

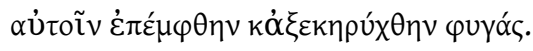

[...] oi $\delta$ ' $\dot{\pi} \pi \omega \varphi \varepsilon \lambda \varepsilon \tilde{v} v$,

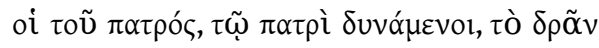

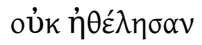

Veuillent les dieux ne jamais l'éteindre cette discorde providentielle! Et puissé-je

demeurer l'arbitre du combat qui affronte les deux frères! Il régnera peu de temps, 
celui qui trône et tient le sceptre; il ne trouvera plus sa place au foyer, celui qui a choisi l'exil, puisque, ni l'un ni l'autre, ils n'ont retenu et protégé l'auteur de leurs jours, lorsqu'il fut ignominieusement expulsé de sa patrie. Oui, si j'ai été jeté à la rue, si j'ai été décrété de bannissement, c'est leur faute. [...] Les fils de mon sang, qui d'un mot pouvaient me sauver, ne daignèrent pas ouvrir la bouche ${ }^{16}$.

C'est entre les deux pièces de Sophocle que furent données les Phéniciennes d'Euripide (en 408) qui fait allusion à cette malédiction à de nombreuses reprises ${ }^{17}$. Après les Tragiques, le Pseudo-Platon, auteur du Second Alcibiade (l'auteur de ce dialogue est probablement un philosophe de l'Académie qui écrivait à la fin du $\mathrm{IV}^{\mathrm{e}}$ ou au cours du III ${ }^{\mathrm{e}}$ siècle) en fait deux fois mention :

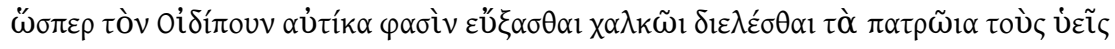
Ainsi, par exemple, Edipe, comme on le raconte, pria [les dieux] de laisser ses fils trancher par l'épée la question de l'héritage paternel ;

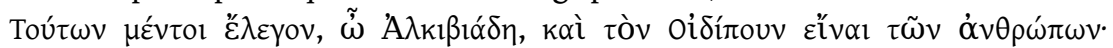

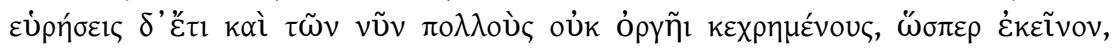

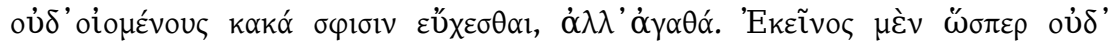

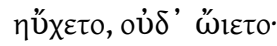

C'est précisément parmi ces gens-là, Alcibiade, que je comptais Edipe. Et tu en trouveras encore aujourd'hui beaucoup qui, sans être en fureur comme lui, ne croient pas demander pour eux, dans leurs prières des maux, mais des biens. Lui, il ne demandait pas des biens, mais il ne croyait pas non plus en demander ${ }^{18}$.

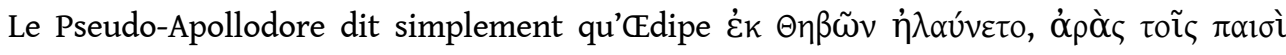

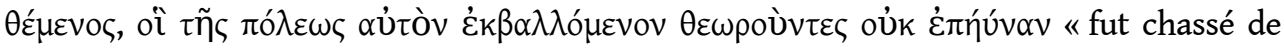
Thèbes, dont il partit en maudissant ses fils, qui assistaient à son expulsion sans le défendre $»^{19}$.

\section{Thésée}

Plus célèbre encore, à notre époque du moins, que la malédiction lancée par đEdipe, est celle appelée par Thésée sur son fils. Chacun connaît, en effet, grâce à Jean Racine, la funeste et vaine passion que le «superbe Hippolyte » fit naître involontairement dans le cœur de Phèdre, sa marâtre, et comment celle-ci, après avoir avoué « ce que jamais on ne devait entendre ", le calomnia auprès de Thésée, de telle sorte que la malédiction lancée par celui-ci causât la mort du jeune homme. Constituée sans doute dès le viI ${ }^{\mathrm{e}}$ siècle, cette légende reprise par les Tragiques, devait connaître un très grand succès, de l'époque hellénistique jusqu'au siècle dernier (G. d'Annunzio), en passant par la littérature romaine (Sénèque et Ovide) et l'époque moderne (R. Garnier en 1573, J. Racine en 1677, J.$\mathrm{Ph}$. Rameau en 1733 pour nous en tenir aux plus célèbres adaptations) ${ }^{20}$. La légende de Thésée est ancienne (Hésiode et les Chants Cypriens la mentionnent) ${ }^{21}$ et celle d'Hippolyte ${ }^{22}$ , qui lui est intimement liée, bien que n'ayant pas connu la même fortune à l'époque archaïque, l'est aussi. La Bibliothèque du Pseudo-Apollodore nous apprend en effet

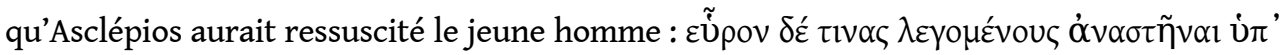

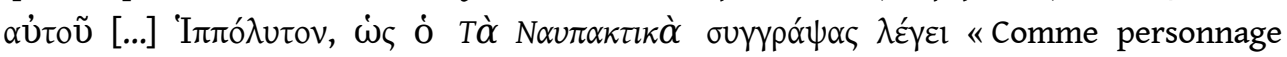
ressuscité par lui je trouve [...] Hippolyte cité par l'auteur des Chants de Naupacte »23. Ce

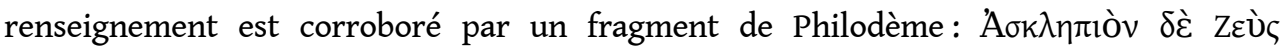

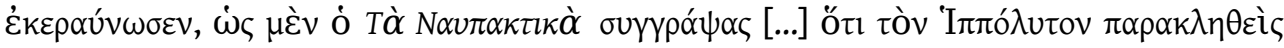

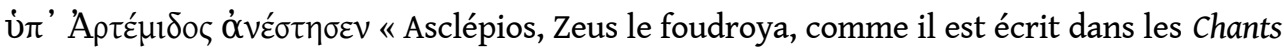
de Naupacte [...] parce qu'il avait ressucité Hippolyte à la prière d'Artémis ${ }^{24}$. Or, Les Chants de Naupacte, attribués à Carcinos et consacrés aux femmes célèbres, s'il faut en 
croire Pausanias ${ }^{25}$, sont généralement datés du $\mathrm{VII}^{\mathrm{e}}$ siècle. Louis Séchan reconstitue ainsi la genèse du mythe :

Si l'on admet que les Chants de Naupacte unissaient déjà Hippolyte à Phèdre et Thésée, les premières années $d u$ VII $^{e}$ siècle marqueraient le dernier terme de l'élaboration de la « nouvelle ». Mais cette date nous parait plutôt correspondre au point initial de sa formation; il est en tout cas probable que les principaux détails de l'histoire humaine d'Hippolyte furent coordonnés vers le début du $\mathrm{vI}^{\mathrm{e}}$ siècle dans une œuvre poétique trézénienne qui inspirera Polygnote et les tragiques athéniens 26 .

10 Mais, en l'état actuel de notre documentation, pour ce qui concerne plus précisément la légende de la passion de Phèdre pour son beau-fils, qui seule nous intéresse du point de vue de la malédiction paternelle, rien n'apparaît avant le début du $\mathrm{v}^{\mathrm{e}}$ siècle. Il semblerait, en effet, s'il faut en croire encore Pausanias, qu'une peinture, datée de 480-476 et ornant la Leschè des Cnidiens de Delphes, la Nékyia de Polygnote, représentait Phèdre parmi les femmes coupables, assise sur une balançoire ${ }^{27}$. L'histoire a été traitée par les Tragiques. Euripide, en particulier, en a fait le sujet de deux de ses pièces. La première, Hippolyte voilé (titre qui n'est pas d'Euripide), datée peut-être de 432-430, est perdue à l'exception d'une vingtaine de fragments ${ }^{28}$; la seconde est celle de 428 qui est conservée. Sophocle, de son côté, a donné, entre les deux Hippolyte d'Euripide, selon l'hypothèse de William Spencer Barrett ${ }^{29}$, une Phèdre, dont il reste quelques fragments ${ }^{30}$. Nous savons également par la Souda que Lycophron avait écrit un Hippolyte; mais aucune trace de son contenu ne s'est conservée ${ }^{31}$.

\section{Amyntor}

11 La dernière malédiction qui va retenir notre attention, n'est pas sans rapport avec celle lancée par Thésée. Dans l'Iliade, le personnage de Phénix raconte comment il a fui le royaume paternel, coupable d'avoir séduit la maîtresse de son père Amyntor :

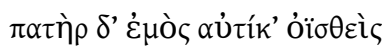

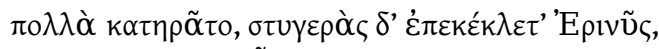

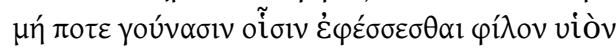

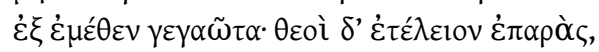

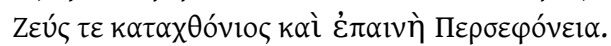

Mais mon père fut prompt à s'en rendre compte. Alors, lançant sur moi force imprécations, il invoquait les Érinyes cruelles : il voulait n'avoir jamais à asseoir sur ses genoux un enfant issu de moi ; et les dieux ont réalisé ses imprécations, Zeus infernal aussi bien que la féroce Perséphone ${ }^{32}$.

C'est à peu près la même histoire que racontent les épigrammes de Cyziques rassemblées dans l'Anthologie Palatine mais il n'y est pas question de malédiction ${ }^{33}$. Les Tragiques se sont emparés de cette histoire. Sophocle, Euripide et Ion composèrent des pièces intitulées Phénix. Seuls quelques fragments de celui d'Euripide en sont conservés ${ }^{34}$. L'histoire y parait traitée d'une manière différente, puisque, semble-t-il, Euripide montrait le jeune homme accusé faussement, ce qui était une innovation par rapport à l' Iliade, et son sort était également différent : il était aveuglé sur l'ordre de son père ${ }^{35}$. 


\section{Les malédictions paternelles lancées par Fidipe, Thésée et Amyntor : analyse}

13 Venons-en, à présent, aux enseignements que nous pouvons tirer de ces récits. Ces malédictions mettent en scène trois acteurs (un destinateur, un médiateur et un destinataire) ainsi qu'un motif qui sert de justification et un résultat. Laissons de côté, pour l'instant, les acteurs et voyons ce qu'il en est des motifs et des effets de la malédiction.

\section{Motifs}

Dans le cas de Thésée et d'Amyntor, les raisons de lancer une malédiction sont graves puisqu'il s'agit de punir une tentative de viol ou un adultère au sein même de l'oikos. Il importe peu à notre propos, dans l'immédiat, que, dans un cas, Hippolyte soit innocent de ce dont l'accuse Phèdre et que, dans l'autre, pour la version homérique qui seule nous intéresse, Phénix soit bel et bien coupable comme il l'avoue lui-même, même s'il a des circonstances atténuantes (il semblerait en effet qu'il n'ait pas rencontré chez sa bellemère une farouche résistance s'il faut en croire Eustathe ${ }^{36}$; par ailleurs, il agissait en service commandé, si l'on peut dire, puisqu'il répondait ainsi aux sollicitations pressantes de sa mère dont il vengeait l'affront ${ }^{37}$ ). Innocent ou coupable, le fils est passé aux yeux de son père pour coupable, et gravement coupable. Gageons que le public destinataire de ces mythes, qu'il s'agisse des auditeurs de l'aède ou du rhapsode, ou des spectateurs athéniens aux Dionysies, ne devait guère être offusqué de voir un père lancer des imprécations contre son fils pour un tel crime. Dans le cas d'ÆEdipe, l'affaire est moins claire. Au fil des récits, les motifs varient. Reprenons les textes dans leur ordre chronologique. Dans la Thébaïde, il est manifestement question d'une désobéissance (l'utilisation de la vaisselle de Cadmos et de Laios : avant de citer le fragment 2, notre source, Athénée, prend en effet le soin de nous donner un résumé de la situation d'où il ressort qu'ÆEdipe avait expressément défendu l'utilisation de ces objets qu'il avait reçus de son père) et/ou d'un affront (la mauvaise portion de viande après un sacrifice). Que les deux motifs soient des doublons, comme il est toujours possible dans l'épopée, ou qu'ils s'insèrent dans une continuité - l'affront s'ajoutant à la désobéissance et la malédiction se précisant lors du second accès de colère d'ÆEdipe qui passe du souhait que naisse entre ses fils la querelle à celui qu'ils meurent de la main l'un de l'autre - n'est pas sans conséquence. Mais comme il est impossible de trancher, laissons cela et venons-en aux Tragiques. Chez Eschyle, la malédiction figurait sans doute, nous l'avons vu, dans l'CEdipe, deuxième pièce de la trilogie, qui est perdue. Il faut donc nous en tenir à ce que nous avons, c'est-à-dire à la cinquième antistrophe du deuxième stasimon des Sept, dont le texte n'est malheureusement pas entièrement assuré :

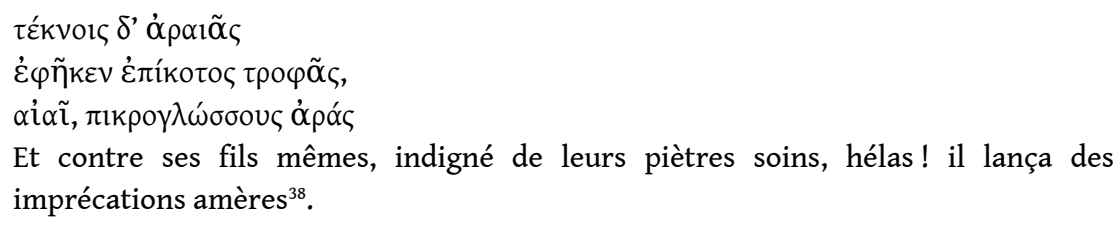

15 La lecture qu'en proposent notamment George Manton et Hanna Roisman ${ }^{39}$ est que la malédiction lancée par ÆEdipe a pour motif l'anagnorisis de son origine et de son inceste : il 
anéantirait ses fils comme il crève ses yeux pour se punir lui-même et pour punir sa descendance. Mais, outre qu'on ne comprend pas pourquoi punir une descendance qui n'est que le fruit et non la cause de l'inceste, quelle pourrait être l'utilité d'ajouter une souillure à une autre en requérant le fratricide? Pourquoi, enfin, ne pas maudire aussi Antigone et Ismène? Nous préférons retenir ce que dit plus explicitement le texte: la malédiction est liée à un défaut de trophè, interprétation retenue par la traduction de Paul Mazon et qui d'ailleurs ne souffrirait guère de discussion... si la lecture des manuscrits était assurée. Malheureusement (ou heureusement, si l'on considère le nombre de philologues qui ont fait de ces vers leurs délices), la leçon n'est sûre ni pour $\dot{\alpha}_{\rho} \alpha \tilde{\alpha}_{\boldsymbol{\alpha}}$, , ni

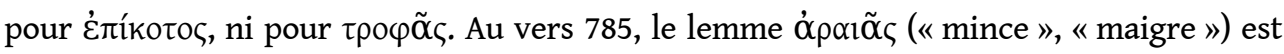

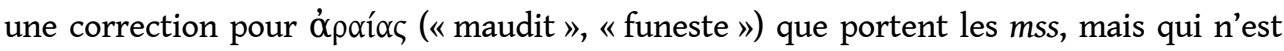
métriquement pas possible et de plus redondant avec ả pó ( "malédictions ») du v. 787; mais Ülrich von Wilamowitz et Carl Robert proposaient pour leur part $\alpha$ $\rho x \alpha i ́ \alpha \varsigma^{40}$. Quant à

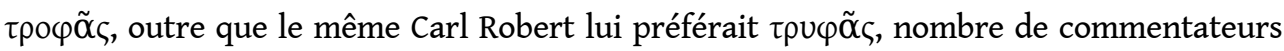
l'interprètent non dans le sens de « soins " mais dans celui de " génération ", c'est-à-dire

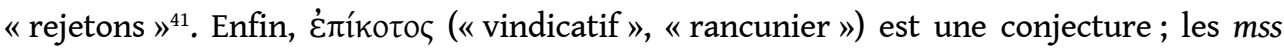
portent Éđíkotous, ce qui ne saurait évidemment s'appliquer au sujet proférant. On pourrait donc aussi bien comprendre ces vers de la sorte : «Et sur ses fils il lança, hélas, de sa langue amère, de funestes malédictions irritées contre sa descendance », comme le propose A. Moreau. Voyons à présent ce qu'il en est chez Euripide. Dans le prologue, Jocaste expose que, depuis qu'ils sont parvenus à l'adolescence, Étéocle et Polynice tiennent leur père aveugle enfermé, espérant ainsi faire oublier la honte et la souillure

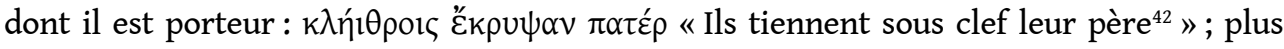
tard, Tirésias rappelle les faits et désapprouve la conduite des fils qu'il qualifie

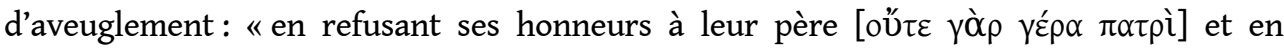
l'empêchant de sortir, ils exaspérèrent un infortuné; et il exhala contre eux des

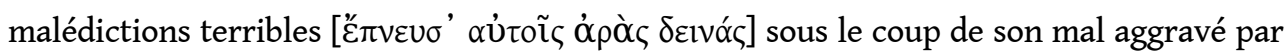
l'outrage ${ }^{43}$ ". Chez Sophocle, enfin, CEdipe est exilé du pays et en fait reproche à Polynice venu requérir son aide : «Dis-moi, misérable, quand tu étais en possession du trône et du sceptre par lesquels ton frère règne aujourd'hui dans Thèbes, n'as-tu pas chassé ton propre père ? [...] Tu m'as voué à la misère, tu m'as jeté dehors ; c'est ta faute, s'il me faut mendier ma nourriture au jour le jour $\aleph^{44}$. On le voit, selon la plupart des versions de la malédiction lancée par đEdipe, il s'agit d'un motif en rapport avec un manquement grave à la trophè due au père ou plus généralement de mauvais soins. Nous en devons déduire, nous semble-t-il, que dans les mentalités des Grecs de l'époque préclassique qui a vu naître ces mythes, déposséder son père du pouvoir ou enfreindre la gérotrophia sont des fautes d'une gravité telle qu'elles méritent la mort, alors que la législation athénienne ne

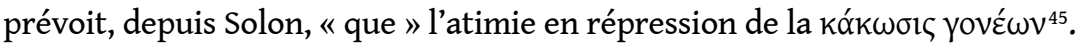

\section{Effets}

16 Quels sont les effets de la malédiction paternelle? Ce qui apparait d'emblée, c'est son efficacité que nous qualifierions volontiers d'exhorbitante. Hippolyte, Étéocle et Polynice meurent comme promis ; Phénix, pour sa part, ainsi que l'a souhaité Amyntor, s'éteindra sans enfant, puisqu'il est déjà un vieillard apais quand il raconte son sort à Achille. Mais la malédiction paternelle n'est pas seulement efficace, elle l'est de façon inexorable. C'est visible, nous semble-t-il, dans la vanité des efforts pour s'y soustraire, particulièrement 
perceptible dans le cas des fils d'Ædipe. Plusieurs sources, en effet, montrent des tentatives d'empêcher la réalisation de la malédiction. Dans un fragment d'un poème, dont l'auteur est très vraisemblablement Stésichore et qui nous a été transmis par le Papyrus de Lille, on voit la mère des deux garçons s'évertuer à trouver une solution pour déjouer la malédiction qui pèse sur eux et dont la réalisation est imminente aux dires de Tirésias : elle propose qu'un tirage au sort détermine lequel des deux détiendra la royauté et lequel s'exilera, avec tout l'or et les autres possessions d'Edipe en guise de compensation ${ }^{46}$. Dans les Sept contre Thèbes, nous apprenons par un messager que Polynice reproche à son frère de l'avoir exilée ${ }^{47}$, ce qui peut être interprété comme une tentative de déjouer la malédiction. En effet, comme le remarque Timothy Gantz, «bien qu'Étéocle soit, depuis le début, conscient de la malédiction prononcée par son père contre lui et son frère, il semble pendant une grande partie de la pièce avoir bon espoir que cette malédiction ait été détournée. Ce n'est qu'au moment où il découvre que Polynice lui fera face à la septième porte que son espérance chancelle ${ }^{48}$ ", et même s'effondre, d'une façon si brutale que certains commentateurs, tel Friedrich Solmsen, ont voulu l'expliquer par un accès de folie ${ }^{49}$. Il est aussi fait allusion à un rêve dans lequel il était question d'un médiateur étranger venant faire la distribution des parts pour entraver l'accomplissement de la malédiction ${ }^{50}$.

17 Cette efficacité est d'autant plus frappante qu'on la voit à l'œuvre même lorsque la malédiction est manifestement injuste. Que l'on songe, par exemple à l'inconséquence qui, dans le fragment 2 de la Thébaïde, fait s'abattre sur les deux fils la punition d'un seul coupable, Polynice ${ }^{51}$. Le caractère injuste de la malédiction lancée par Thésée est souligné

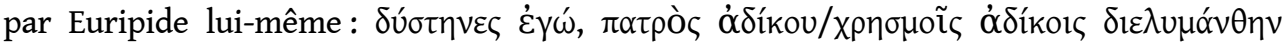
"Ah! Malheureux! L'inique arrêt d'un père inique a consommé ma ruine » affirme Hippolyte mourant ${ }^{52}$. Et Artémis semble attester de la culpabilité de Thésée : $\dot{\alpha} \lambda \lambda \grave{\alpha}$

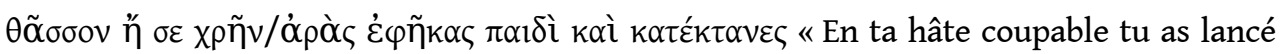
l'imprécation contre ton fils, et tu l'as tué » dit-elle en s'adressant au roi ${ }^{53}$. Enfin, il faut insister sur le fait que, si le sort d'un Phénix (l'exil et la mort sans postérité) peut paraître proportionné à sa faute bien réelle dans une société où l'adultère était sévèrement puni, dans le cas des enfants d'€Edipe, la malédiction peut paraître disproportionnée. C'est ainsi qu'un exégète récemment disparu a pris pour point de départ de son enquête sur đEdipe son «insatisfaction causée par les fragments deux et trois de la Thébaïde», où la « disproportion entre le caractère véniel des fautes commises par les fils d'ÆEdipe et l'horreur des imprécations $»^{54}$ le choque. Il a supposé, en s'appuyant sur une étude comparative, que le véritable motif était un acte d'anthropophagie. Au vrai, ce sentiment de disproportion ne heurte pas que les sensibilités modernes. Dès l'Antiquité, nombre de commentateurs l'ont éprouvé et ont cherché à expliquer cette disproportion. Ainsi le scholiaste A de l'Iliade (codex de Villoison du $\mathrm{X}^{\mathrm{e}}$ s.- $\mathrm{XI}^{\mathrm{e}} \mathrm{s}$. = Venet. Marc. 454) suppose-t-il qu'œdipe aurait eu un motif plus sérieux : ses fils auraient été calomniés auprès de leur père par Astydaméia leur belle-mère, ce qui revient à placer la malédiction du roi thébain au niveau de celles lancées par Thésée ou Amyntor ${ }^{55}$. D'autres ont imputé cette malédiction à un accès de démence d'ÆEdipe, comme d'ailleurs y invite Eschyle lui-même

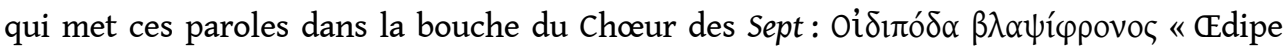
dont la raison est atteinte $\Perp^{56}$. C'est encore l'avis de l'auteur du Second Alcibiade : A $\lambda \lambda \grave{\alpha} \sigma \mathrm{v̀}$

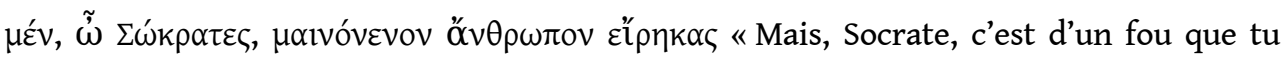
parles ${ }^{57}$. Quand on ne taxe pas Edipe de démence, on n'en critique pas moins son

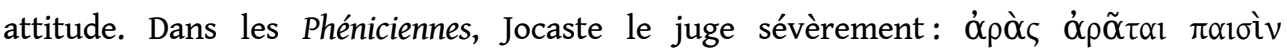




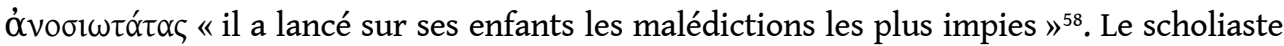
de l'ÆEdipe à Colone parait également choqué par cette malédiction puisqu'il estime

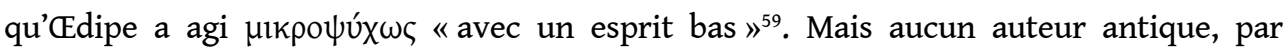
contre, ne s'étonne de l'efficacité des imprécations paternelles.

\section{Médiateur}

C'est ici qu'il faut revenir, pour terminer, aux acteurs de la malédiction et en particulier au médiateur qui se charge de l'exécuter. Il s'agit d'une divinité, ce qui contribue à expliquer son efficacité. Dans les textes les plus anciens (Homère et le fragment 3 de la Thébaïde) il s'agit de Zeus (Zeus Infernal dans l'Iliade, en association avec Perséphone). Mais les imprécations elles-mêmes ont été divinisées puisqu'il existait un sanctuaire des Arai à Athènes, comme nous l'apprend Hésychius ${ }^{60}$. Cette divinisation des arai apparaît pour la première fois dans les mythes chez les Tragiques ${ }^{61}$. Dès Eschyle, Ara est, à n'en pas douter, une divinité, comme le montre le vers 70 des Sept où Étéocle évoque Apó $\tau$ ' 'E

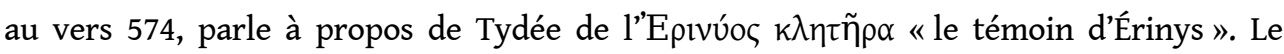

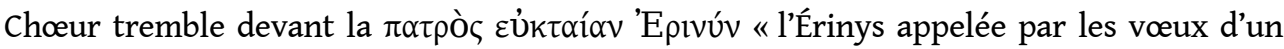
père " (v. 723) et semble faire de celle-là l'exécutrice de ceux-ci (cf. les v. 785-791).

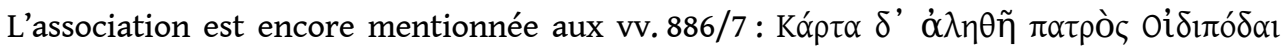

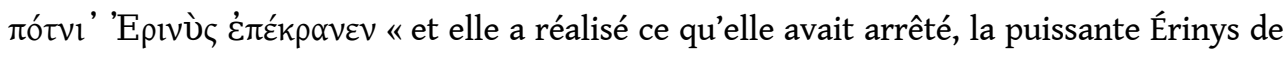
leur père đEdipe!» L'Ara se confond donc avec Érinys ou, pour le moins est mise en œuvre par elle ${ }^{63}$. Ceci n'est pas surprenant étant donné qu'Érinys est ordinairement chargée de punir les offenses et les crimes commis contre les parents, qui semblent, dès Homère, placés sur le même plan que ceux commis contre les dieux ${ }^{64}$. C'est cette ressemblance entre les Arai et Érinys qui rend plus opérant le vœu paternel et plus rapide

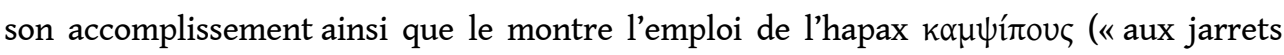
souples ») du vers 791 qui, comme le remarquent Liana Lupas et Zoe Petre, " évoque la monstruosité et la vitesse miraculeuse de l'Érinys ${ }^{65}$ \%. Roger Vallois, étudiant des attestations d'Arai, remarque que "par certains caractères, l'ơoó ressemble à l'Érinye. Elle agit sur le sol, sur tout ce qui en naît ou en vit; elle veille sur les tombeaux; elle défend les dernières volontés des morts; elle tue et détruit; c'est une puissance chthonienne, infernale et vengeresse »; mais il les distingue pourtant : «L'ó $\rho \alpha ́$, produit d'une parole vivante, est plus souple, plus diverse et, si l'on peut dire, plus intelligente que l'Érinye. Elle tient compte des volontés ${ }^{66} »$. La mise en relation entre Ara et Érinys n'est pas le propre d'Eschyle mais, en ce qui concerne la malédiction lancée par đEdipe, c'est chez lui que l'articulation apparait le plus nettement. Eschyle y portait d'ailleurs un intérêt certain, comme en témoigne l'Orestie, où Ara et Érinyes sont également encore confondues ${ }^{67}$. Sophocle les distingue et même les hiérarchise, en plaçant « au-dessus des Érinyes sa rótvl' Apó, personnification de la justice immanente, sorte de LoiImprécation » comme le note Roger Vallois ${ }^{68}$. Euripide interprète différemment l'ara ${ }^{69}$. S'il mentionne l'Érinys paternelle aux vers 253 et 623, ce n'est jamais en liaison avec l'ara qui, du reste, ne semble pas, ici, divinisée. Il est en revanche question chez lui du « démon vengeur » d'ÆEdipe aux vers 1556-1559:

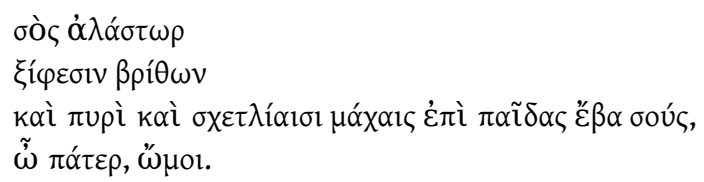



prérogatives, qui met la malédiction en branle, que celle-ci est si redoutable, comme si les
dieux ne pouvaient rester sourds aux imprécations quand elles sont sollicitées au nom de la puissance paternelle. Platon, du reste, ne dit pas autre chose dans les Lois :

oỉír

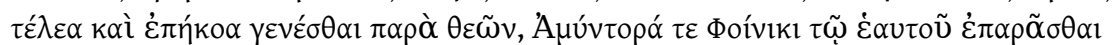

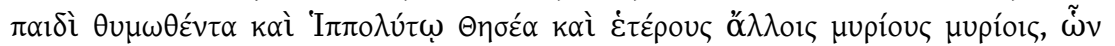

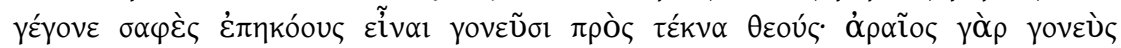

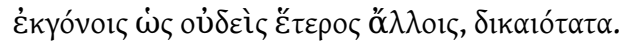

๔dipe, à ce qu'on raconte, sur ses enfants qui le méprisaient, fit des imprécations que les dieux exaucèrent avec une plénitude dont témoigne la tradition universelle. Amyntor, dans sa colère, appela sur son fils Phœnix, Thésée sur son fils Hippolyte, et combien d'autres pères sur combien d'autres fils, des malheurs qui montrèrent clairement comment les dieux exaucent les prières des pères contre leur fils, car la malédiction d'un père contre ses enfants a plus d'effet que n'importe quelle autre contre n'importe qui, et c'est toute justice ${ }^{75}$. 


\section{Sources et commentaires}

Barrett W. S. (1964), Euripides Hippolytos, Edited with Introduction and Commentary, Oxford.

Davies M. (1988), Epicorum Grcecorum Fragmenta, Göttingen.

Garvie A. F. (1969), Aeschylus'Supplices. Play and Trilogy, Cambridge.

Halleran M. R. (1995), Euripides'Hippolytus. With Introduction, Translation and Commentary, Warminster.

Hutchinson G. O. (1985), Aeschylus, Septem contra Thebas, Oxford.

Lupas L. et Petre Z. (1981), Commentaire aux Sept contre Thèbes d'Eschyle, Paris-Bucarest.

Nauck A. (1889), Tragicorum Grcecorum Fragmenta, Leipzig, (rééd. et suppl. par B. Snell, Hidelsheim, G. Olms, 1964) $)^{3}$.

Parsons P. J. (1977), « The Lille Stesichorus », ZPE 26, p. 7-36.

Rose H. J. (1957-1958), A Commentary of the Surviving Plays of Æschylus, Amsterdam.

Webster Th. B. L. (1967), The Tragedies of Euripides, Londres.

\section{Études}

Baldry H. C. (1956), « The Dramatization of the Theban Legend », G\&R 3, p. 25-27.

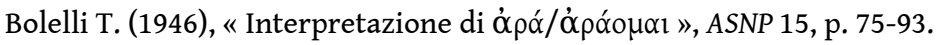

Burkert W. (1981), « Seven against Thebes : an oral tradition between Babylonian magic and Greek litterature », dans Brillante C., Cantilena M. et Pavese C. O. (dir.), I Poemi epici, rapsodici non omerici e la tradizione orale, Padoue, p. 29-51.

Burnett A. P. (1973), « Curse and Dream in Aeschylus'Septem », GRBS 14, p. 343-368.

Cameron H. D. (1964), « The Debt to Earth in the Seven against Thebes », TAPhA 95, p. 1-8.

Cameron H. D. (1971), Studies on the Seven Against Thebes of Aeschylus, Paris-La Haye.

Damet A. (2012), La septième porte. Les conflits familiaux dans l'Athènes classique, Paris.

Daremberg Ch. et Saglio E. (1873), Dictionnaire des Antiquités grecques et romaines, Paris.

Dodds E. R. (1925), « The AI $\Delta \Omega \Sigma$ of Phaedra and the meaning of the Hippolytus », CR 49, p. 102-104.

Dodds E. R. (1977), Les Grecs et l'irrationnel, Paris.

Fraser A. D. (1922), « The Ancient Curse : Some Analogies », CJ 17, p. 455-460.

Gantz T. (2004), Mythes de la Grèce archaïque, Paris.

Harrisson A. R. W. (1968), The Law of Athens, I, The Family and Property, Oxford.

Jouan F. (1989-1990), « Femmes ardentes et chastes héros chez Euripide », SEJG 31, p. 187-208.

Jouanna J. (2007), Sophocle, Paris.

Judet de La Combe P. (1987), «Étéocle interprète. Action et langage dans la scène centrale des Sept contre Thèbes d'Eschyle », ELA 3, p. 57-79.

Kakridès J. Th. (1928), « Der Fluch des Theseus im Hippolytos », RhM 87, p. 21-33. 
Karsai G. (1993), « Ædipe et Créon, Thésée et Hippolyte », dans Machin A. et Pernée L. (dir.), Sophocle, le texte, les personnages, Aix-en-Provence, p. 329-342.

Legras L. (1905), Les légendes thébaines dans l'épopée et la tragédie grecques, Paris, thèse complémentaire de la faculté des lettres de l'université de Paris.

Lipsius J. H. (1905-1915), Das Attische Recht und Rechtsverfahren unter Benutzungen des Attischen Prozesses, Leipzig.

Manton G. R. (1961), « The Second Stasimon of the Seven against Thebes », BICS 8, p. 77-84.

Meillet A. (1925), « Remarques sur l'étymologie de quelques mots grecs », BSL 26, p. 19-20.

Moreau A. (1979), « À propos d'ÆEdipe. La liaison entre trois crimes : parricide, inceste et cannibalisme », ELA I, p. 97-127.

Moreau A. (1976), « Fonction du personnage d'Amphiaraos dans les Sept contre Thèbes : le "blason en abyme" ", BAGB, p. 158-181.

Parker R. (1983), Miasma. Pollution and Purification in Early Greek Religion, Oxford.

Pellizer E. (1982), « Cacciatori neri e virgini orse », Pellizer E., Favole d'identità, favole di paura. Storie di caccia e altri racconti della Grecia antica, Rome, p. 11-71.

Picard Ch. (1928), « "Phèdre” à la balançoire et le symbolisme des pendaisons ", RA 28, p. 47-64.

Podlecki A. J. (1964), « The character of Eteocles in Aeschylus'Septem », TAPhA 95, p. 283-299.

Robert C. (1915), Oidipus, Geschichte eines poetischen Stoffes im Griechischen Altertum, Berlin.

Roisman H. M. (1988), « Oedipus'Curse in Aeschylus'Septem », Eranos 56, p. 77-84.

Rosenmeyer Th. G. (1952), « The Wrath of Oedipus », Phoenix 6, p. 92-112.

Saïd S. (1978), La faute tragique, Paris.

Scotto di Carlo J., « The House of Labdacus vs. the House of Atreus. Use of the curse by Sophocles and Aeschylus », CB 54, 1977, p. 10-14.

Séchan L. (1911), « La légende d'Hippolyte dans l'Antiquité », REG 24, p. 105-151.

Segal Ch. (1972), « Curse and Oath in Euripides'Hippolytus », Ramus I, p. 165-180.

Segal Ch. (1987), La musique du sphinx, Paris.

Séveryns A. (1928), Le cycle épique dans l'école d'Aristarque, Liège-Paris.

Sewell-Rutter N. J. (2007), Guilt by Descent. Moral Inheritance and Decision Making in Greek Tragedy, Oxford.

Smith O. L. (1969), «The Father's Curse. Some Thoughts on the Seven Against Thebes », C\&M 30, p. 27-43.

Solmsen F. (1937), « The Erinys in Aischylos'Septem », TAPhA 68, p. 197-211.

Sutton D. F. (1978), « Euripides'Theseus », Hermes 106, p. 49-53.

Tschiedel H. J. (1969), Phodra und Hippolytus. Variationen eines tragischen Konfliktes, Erlangen.

Vallois R. (1914), «’APAI », BCH 38, p. 250-271.

Wick Cl. (2003), « Le tirage au sort : un "leitmotiv" dans la "Thébaïde de Lille" et les Sept contre Thèbes ", Museum Helveticum LX-3, 2003, p. 167-174.

Wilamowitz von Ü. (1921), Griechische Verskunst, Berlin. 


\section{NOTES}

1. Voir, par exemple, le Trésor de la Langue Française, s.v. « malédiction ».

2. Meillet 1925, p. 19-20 et Bolelli 1946, p. 75-93.

3. Baldry 1956.

4. Sur ces deux poèmes, voir Robert 1915, p. 144 sq. et 169 sq. Voir aussi Legras 1905, p. 50-87 et Séveryns 1928, p. 211-223.

5. "Le plus ancien et le plus beau poème du Cycle après l' Iliade et l'Odyssée " de l'avis de Séveryns (1928, p. 211).

6. Burkert 1981, p. 34 : la Thébaïde serait centrée sur les fils d'œEdipe.

7. Voir Legras 1905, p. 37-38 et Séveryns 1928, p. 212.

8. Thébaïde, fr. 2 (Davis EGF) = Athénée, XI, 465e (trad. Legras 1905, p. 32, légèrement modifiée).

9. Thébaïde, fr. 3 (Davis EGF) = schol. Laur. in Sophocle, Edipe Roi, v. 1375 (trad. Moreau 1979, p. 98).

10. Voir Baldry 1956, p. 28-29.

11. Pour un essai de reconstitution, principalement à partir des fables d'Hygin, voir Robert 1915, p. 274-283. Voir également le commentaire de la pièce par Lupas et Petre 1981.

12. Voir Baldry 1956, p. 30-32 et Lupas et Petre 1981, p. 4.

13. Eschyle, Sept contre Thèbes, v. 710-711. Sauf mention contraire la traduction retenue est celle procurée par la Collection des Universités de France («Budé»). Voir Manton 196, p. 78-79; Burnett 1973, p. 343-368 et Judet de La Combe 1987, p. 75, n. 5.

14. Vers 70, 574, 652-655, 695-697, 709, 720-726, 785, 819, 832-833, 886-887 et 898.

15. Solmsen 1937, p. 197-211, en particulier p. 199 sq.

16. Sophocle, Edipe à Colone, v. 421-430 et 441-443.

17. Aux v. 63-68, 253-55, 334, 623, 765, 876-877, 1425-1426, 1556-1559 et 1610-1611.

18. [Platon], Second Alcibiade, $138 \mathrm{c}$ et $141 \mathrm{a}$.

19. [Apollodore], III, 5, 9 (trad. J.-C. Carrière).

20. Voir Tschiedel 1969, p. 57-99.

21. Voir Séchan 1911, notamment p. 105-106.

22. Il est possible qu'Hippolyte ait été d'abord une divinité courotrophe. Sur ses caractéristiques de "chasseur noir" voir Pellizer 1982, p. 22 sq.

23. [Apollodore], III, 10, 3.

24. Philodème, Péri eusébéias, 131, 11. 5-8 et 11-15 (éd. Gomperz p. 52), trad. J.-B. Bonnard.

25. Pausanias, $X, 38,6$.

26. Séchan 1911, p. 135.

27. Pausanias, X, 29, 3-4. Voir Picard 1928, p. 47-64.

28. TGrF Nauck ${ }^{2}$, fr. $428-448$.

29. Barret 1992, p. 12-14.

30. TGrF Nauck ${ }^{2}$, fr. 616-634.

31. Souda, s.v. " $\Lambda$ кó $\varphi \rho \omega v ", \lambda 827=T G r F, \mathrm{I}, 100 \mathrm{~T} 3$ et $\mathrm{F} 1 \mathrm{~g}$.

32. Homère, Iliade, IX, 453-57.

33. Cf. Anthologie Palatine, III, 3.

34. Euripide, fr. 804-818 TGrF Nauck ${ }^{2}$. Sur le Phénix de Sophocle, voir Jouanna 2007, p. 672-673 et Damet 2012, p. 104-105.

35. Cf. Aristophane, Acharniens, v. 421 ; Ménandre, Samienne, v. 671-672 ; [Apollodore], III, 13, 8.

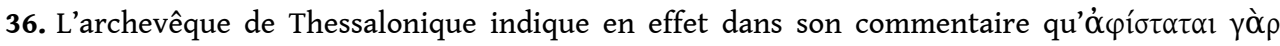

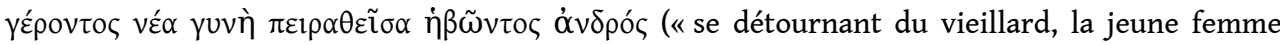
provoquait le jeune homme »), Eustathe, ad Homeri Iliadem, IX, v. 448 (trad. J.-B. Bonnard). 
37. Cf. Homère, Iliade, IX, 447 sq.

38. Eschyle, Sept contre Thèbes, v. 785-787.

39. Voir Manton 1961, p. 77-84 et Roisman 1988, p. 77-84

40. Wilamowitz 1921, p. 203-204 ; Robert 1915, p. 263 sq.

41. Ainsi font Baldry, p. 31-32 (après Hermann, Schütz et Weill) ; Manton 1961, p. 82 ; Burnett, p. 354 ; Moreau 1976 ; Hutchinson 1985 ; Roisman 1988.

42. Euripide, Phéniciennes, v. 64 (trad. J.-B. Bonnard).

43. Euripide, Phéniciennes, v. 874-877.

44. Sophocle, ÆEdipe Roi, v. 1354 sq. (trad. R. Pignarre).

45. Cf. Lysias, XXXI, Contre Philon, § 21 ; Eschine, I, Contre Timarque, § 28 ; Démosthène, XXIV, Contre Timocrate, § 107 ; Isée, VIII, Sur la succession de Kiron, § 32 ; et la loi de Solon apud Diogène Laërce, I, 2, 55. Voir Lipsius 1905-1915, p. 343-344 ; Harrisson 1968, p. 77-78 et, en dernier lieu Damet 2012, p. 211-234.

46. Stésichore, 222b (= Pap. Lille, 76a, b, c) publié par Parsons 1977.

47. Cf. Eschyle, Sept contre Thèbes, v. 637-638.

48. Gantz 2004, p. 891.

49. Voir Solmsen 1937.

50. Cf. Eschyle, Sept cintre Thèbes, v. 637-638 et voir Burnett 1973.

51. Voir Smith 1969, p. 31 sq.

52. Euripide, Hippolyte, v. 1348-1349.

53. Ibid., v. 1323-1324.

54. Moreau 1979, p. 97 et 99.

55. Cf. Schol. A ad Homère, Iliade, IV, 376.

56. Eschyle, Sept contre Thèbes, v. 725, (trad. J.-B. Bonnard).

57. [Platon], Alcibiade Mineur, 138b.

58. Euripide, Phéniciennes, v. 67.

59. Schol. Laur. in Sophocle, Edipe Roi, v. 1375.

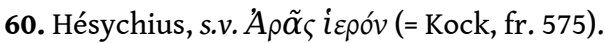

61. Voir Vallois 1914, p. 262.

62. Voir Roscher, Lex., s.v. Apaí.

63. Voir l'étude de Solmsen 1937 et le commentaire de Lupas et Petre 1981, p. 36-37.

64. Voir Saïd 1978, p. 296 et cf. Hésiode, Travaux et Jours, v. 327 sq. où l'on voit un exemple d'équivalence entre outrage aux parents et outrage aux dieux, ce que Socrate (Xénophon, Mémorables, IV, 4, 19-20) appelle enfreindre les lois non écrites.

65. Lupas et Petre 1981, p. 246.

66. Vallois 1914, p. 257.

67. Cf. Eschyle, Choéphores, v. 402-406 et Euménides, v. 417.

68. Vallois 1914, p. 260.

69. Pour une comparaison des arai dans les Sept et dans les Phéniciennes, voir Solmsen 1937, p. 209-211.

70. Euripide, Phéniciennes, v. 1556-1558

71. Solmsen 1937, p. 208. Voir Dodds 1977, chap. II et notamment p. 55 sq. et Parker 1983, chap. VI.

72. Daremberg Ch. et Saglio E., DA, s.v. Devotio, p. 113 b (Bouché-Leclercq A.).

73. Euripide, Hippolyte, v. 1028-1031 (trad. M. Delcourt-Curvers). Sur ce redoublement et son ironie tragique, voir Segal 1987 (chapitre «Penthée et Hippolyte sur le divan et sur la grille: Lecture psychanalytique et lecture structuraliste de la tragédie grecque ", notamment p. 168-169).

74. Segal 1987, p. 153.

75. Platon, Lois, 931 b-c. 


\section{RÉSUMÉS}

Les poèmes du Cycle épique (Edipodie, Thébaïde) et les tragédies grecques classiques (Eschyle, Sophocle, Euripide) permettent l'étude des formes et de l'expression de la malédiction paternelle dans les mythes grecs, à partir des exemples d'œdipe, Thésée et Amyntor.

From the poems of the Epic Cycle (Oedipodia, Thebaid) and the Greek tragedies (Aeschylus, Sophocles and Euripides), this article deals with the forms and the expression of the paternal curse in the Greek myths through the examples of Oedipus, Theseus and Amyntor.

\section{INDEX}

Mots-clés : malédiction, père, cycle épique, Eschyle, Sophocle, Euripide, đdipe, Thesée, Amyntor

Keywords : curse, father, epic cycle, Aeschylus, Sophocles, Euripides, Oedipus, Theseus

\section{AUTEUR}

\section{JEAN-BAPTISTE BONNARD}

Université de Caen Basse-Normandie, ANHIMA - UMR8210 